\title{
EXPECTATIVAS DE LOS AGENTES DE DESARROLLO RURAL ARGENTINOS SOBRE LA PSICOLOGÍA Y SOBRE LA INSERCIÓN PROFESIONAL DE LOS PSICÓLOGOS EN EL ÁMBITO DE LA EXTENSIÓN RURAL
}

\section{Argentine RURal deVElopment agents' expectations about Psychology and ABOUT THE EMPLOYABILITY OF PSYCHOLOGISTS IN THE AREA OF RURAL EXTENSION}

\author{
Fernando PABLo LANDINI*
}

\begin{abstract}
*Doctor en Psicología y Magister en Desarrollo Rural y en Desarrollo Local. Becario Postdoctoral del Consejo Nacional de Investigaciones Científicas y Técnicas (CONICET), docente e investigador de la Facultad de Psicología de la Universidad de Buenos Aires (UBA) y de la Universidad de la Cuenca del Plata.E-Mail: Iandini_fer@hotmail.com
\end{abstract}

\section{RESUMEN}

La Psicología posee una importante potencialidad para trabajar en el ámbito de la extensión y el desarrollo rural. No obstante, no existe evidencia empírica suficientemente amplia de las expectativas de los agentes de desarrollo sobre la Psicología. Por este motivo se realizó una investigación transversal de carácter cuantitativo. La muestra fue no probabilística incidental e incluyó participantes de todas las regiones de Argentina. Se encuestó a extensionistas rurales que trabajan en las principales instituciones que realizan $e x-$ tensión rural en el ámbito público. La encuesta, que fue respondida por 219 sujetos $(9.06 \%$ del total nacional) incluyó preguntas cerradas sociodemográficas y abiertas respecto de las expectativas sobre la Psicología. Las preguntas abiertas fueron categorizadas y organizadas con el apoyo de la teoría fundamentada y el software Atlas Ti. Las categorías fueron convertidas en variables para estudiar su relación con las preguntas sociodemográficas con apoyo del software SPSS.

Se concluye que la mayor parte de los extensionistas rurales del ámbito público argentino (un $88 \%$ ) considera que la Psicología podría ayudarlos a resolver algunos de los problemas a los que se enfrentan, por ejemplo, brindando capacitación y asesoramiento a extensionistas rurales así como participando en equipos interdisciplinarios de $e x$ tensión rural. Además, podrían trabajar directamente con productores en la gestión de procesos grupales.

En términos generales, se destaca la potencialidad de la Psicología en el ámbito del trabajo grupal, la comunicación, el apoyo emocional y el cambio de actitudes y prácticas. Finalmente, se destacan las limitaciones de la investigación realizada.

Palabras clave: Agricultura familiar; Desarro1lo; Extensión rural; Interdisciplina; Pequeños productores; Rol profesional.

\section{ABSTRACT}

Many scholars have argued that rural extension and development processes need an interdisciplinary approach, one that includes contributions from different sciences, particularly social ones. In this context, social and community psychology have a great potential to address this topic. In spite of this potentiality, psychology has produced scarce useful contributions to rural development and to extension processes. Additionally, limited empiri- 
cal support has been given to probe this potentiality, which makes this a theoretical argument.

Thus, a cross-sectional, quantitative investigation was conducted in Argentina. An incidental, non-probabilistic sample was used, which included participants from all regions of the country. Rural extension agents working in the National Institute of Agrarian Technology, the ProHuerta Program and the Under secretariat of Family Farming of the Ministry of Agriculture, Husbandry and Fisheries were surveyed via email. Local authorities of these institutions helped the researchers to send and receive the completed surveys in different ways. The reply to the poll was voluntary. 219 completed forms (143 men and 76 women) were received, which represent $9.06 \%$ of rural extensions working in public institutions in $\mathrm{Ar}$ gentina. The survey included socio-demographic, closed questions, and open ones regarding the problems faced by rural extensions in their everyday practice and their expectations about Psychology as a mean to address them. In order to analyze the open questions, different categories of analysis were built and thoroughly described to cope with ambiguous cases, which were used to categorize all replies. In this process, grounded theory and Atlas Ti software were utilized. Next, these categories were transformed into dichotomist variables, expressing the presence of the category presence of the variable and its absence, the absence of the variable. Then all variables were incorporated to SPSS software, which allowed for the quantification of the results and the study of statistical relationships between different variables.

The most important result of this investigation is that $88 \%$ of the Argentine rural extensionists working in the National Institute of Agrarian Technology, the ProHuerta Program and the Under secretariat of Family Farming consider that Psychology can contribute to solve at least some of the problems they face when doing rural extension. Interestingly, this represents a very high and unexpected percentage, given psychology is not a social science traditionally related to rural issues or settings. In consequence, it is clear that there is a potential demand for psychologists in the field of rural development processes, which is not being addressed.

Additionally, the replies allowed for the description of the role psychologists should fulfill when working in this field, from rural extensions' point of view. They argue that psychologists could work with both, them and farmers. On the one hand, they could train, advice and provide with practical tools to rural extensionists. Additionally, they could take part in interdisciplinary rural extension teams. In practical terms, they could contribute in fields such as cooperative processes and group management, design and implementation of projects, understanding of small farmers' rationale, conflict resolution, lack of adoption of technologies, and scarce participation and commitment to development projects. On the other hand, psychologists also could work directly with small farmers, providing training in different topics, and managing groups, particularly in the case of participatory processes. Additionally, they could strengthen small farmers' self-esteem, a problem that rural extensionists related to passive attitudes and fatalism.

To conclude, it is important to underline that, knowing this expected role, psychologists should start a dialogue with rural development agents in order to generate a shared conception of psychologist working in the field of rural extension, which includes both, psychologists' and rural extensionists' point of view.

Key words: Family agriculture; Development; Rural extension; Interdisciplinary; Small farmers; Professional role.

\section{INTRODUCCIÓN}

Diversos autores han señalado la necesidad de abordar de manera interdisciplinaria las tareas de extensión y desarrollo rural (Carballo, 2002; Herrera Tapia, 2006; Méndez Sastoque, 2006; Sánchez Cadena, 2011), incorporando contribuciones provenientes de las ciencias sociales (Landini, Murtagh \& Lacanna, 2009; Machado, Hededüs \& Silveira, 2006; Tsakoumagkos, González \& Román, 2009). Esta postura se basa en el reconocimiento de la complejidad y multidimensionalidad de los procesos de innovación y desarrollo (Klerkx, Aarts \& Leeuwis, 2010; 
Leeuwis, 2004; Leeuwis \& Aarts, 2011), particularmente cuando son pensados más allá de un paradigma productivista (Albuquerque, 1995; Di Pietro, 2001; Landini, 2007a; MaxNeef, Elizalde \& Hopenhayn, 1993).

La extensión rural ha sido definida de múltiples maneras y abordada desde diferentes perspectivas (Leeuwis, 2004). En términos genéricos puede definírsela como el trabajo que realizan agentes de desarrollo, generalmente ingenieros agrónomos y veterinarios (Landini, 2012a), de manera conjunta con productores agropecuarios e incluso con otras instituciones relacionadas, con el fin de facilitar procesos de innovación a nivel productivo, comercial u organizacional orientados a mejorar sus condiciones de vida, hacer más eficiente la producción y/o proteger el medio ambiente. En el contexto del trabajo con pequeños productores, la extensión rural involucra fundamentalmente transferencia de capital por medio de entrega de créditos, subsidios o insumos, capacitación en el ámbito productivo y trabajo grupal o cooperativo, ya sea para favorecer el intercambio de conocimientos, mejorar las posibilidades de comercialización o facilitar el uso conjunto de herramientas y maquinarias.

En su concepción tradicional, la extensión rural era pensada como una relación jerárquica entre técnicos y productores orientada a la transferencia de conocimientos científicos a los productores del campo (De Schutter, 1982) con el fin de modernizar las explotaciones y aumentar la productividad (Alemay \& Sevilla Guzmán, 2007; Carballo, 2002). No obstante, este modelo ha recibido múltiples críticas, referidas a la falta de reconocimiento de la complejidad de los procesos de extensión e innovación (Leeuwis, 2004; Machado, Hededüs \& Silveira, 2006), al énfasis dado a la dimensión informativa en lugar de a la educativa en el trabajo del extensionista (Sánchez Cadena, 2011) y, particularmente en América Latina, a la relación de poder que establece entre ambos actores (Freire, 1973; Schaller, 2006; Valentinuz, 2003). Así, hoy se tiende a pensar la extensión como una tarea centrada en procesos comunicativos, estrategias educativas no formales y actitudes dialógicas y horizontales (Landini, Murtagh \& Lacanna, 2009) por medio de las cuales el extensionista, ocupando el rol de facilitador (Leeuwis, 2004), busca generar conocimiento conjunto (Freire, 1973), aprendizaje social (Leeuwis \& Pyburn, 2002), articulación social e innovación (Leeuwis, 2004).

Vista así, la extensión rural excede en mucho la dimensión puramente técnica y requiere contribuciones de las disciplinas sociales. En este contexto, llama la atención la escasez de aportes de utilidad práctica provenientes de la Psicología (Landini, Benítez \& Murtagh, 2010; Murtagh \& Landini, 2011), razón por la cual se ha argumentado la necesidad de generar una psicología del desarrollo rural (Landini, 2010; Landini, 2011a; Landini \& Bianqui, 2012; Landini, Long, Leeuwis \& Murtagh, 2012) derivada de los lineamientos de la Psicología Comunitaria (Montero, 2004; Sánchez Vidal, 1991). No obstante, y pese a que en un trabajo previo han sido analizadas las contribuciones potenciales de la Psicología a los proyectos de extensión rural en América Latina (Barilari, Landini, Logiovine \& Rotman, 2011), esta propuesta ha permanecido fundamentalmente a nivel especulativo -con la excepción de dos casos de intervención- (Landini, en prensa; Landini, Bianqui \& Russo, 2012), sin que se haya estudiado sistemática y empíricamente ni la existencia de interés de los extensionistas en relación a contribuciones por parte de la Psicología ni sus expectativas concretas. Así, en la investigación que se informa se analizaron las expectativas de los extensionistas rurales con respecto a la Psicología, a fin de brindar evidencia sobre el interés existente en torno a contribuciones de la Psicología al ámbito de la extensión y el desarro110.

\section{Metodología}

Para alcanzar los objetivos propuestos, se realizó un estudio descriptivo de poblaciones de carácter transversal mediante encuestas (Montero \& León, 2007) utilizándose una 
muestra no probabilística incidental (Tomás Sábado, 2009). La encuesta construida para esta investigación ${ }^{1}$, se realizó por correo electrónico a extensionistas rurales que trabajan en las principales instituciones que hacen extensión rural en el ámbito público en Argentina.

En total se obtuvieron 219 respuestas, 143 de hombres y 76 de mujeres: 106 del Instituto Nacional de Tecnología Agropecuaria (INTA), 72 del Programa ProHuerta (implementado conjuntamente por el Ministerio de Desarrollo Social y el INTA) y 41 de la Subsecretaría de Agricultura Familiar (SAF) del Ministerio de Agricultura, Ganadería y Pesca. En el caso de técnicos que trabajaban tanto en ProHuerta como en otras iniciativas de INTA, fueron categorizados como de ProHuerta a aquellos que dedicaban al programa el $50 \%$ o más de su tiempo laboral en la institución. La cantidad total de extensionistas del INTA y ProHuerta, según informó la Coordinación Nacional de Transferencia y Extensión del INTA, es de 1.567 entre ambos, por lo que la muestra representa el $11.36 \%$ del total. En el caso de la SAF cuenta con 1.245 técnicos, de los cuales trabajan en campo entre 800 y 900 . Así, la muestra de la SAF representaría el $4.82 \%$ del total nacional (considerando que son 850 los técnicos que trabajan como extensionistas). En conjunto, la muestra representa aproximadamente el $9.06 \%$ del total.

Para la obtención de las respuestas se contactaron a las autoridades provinciales del ProHuerta, de la SAF y a las coordinaciones territoriales del INTA (Estaciones Experimentales Agropecuarias). Se obtuvo el apoyo de todas las provincias del país. El mismo se concretó de diferentes maneras según el caso. En algunas oportunidades, se acordó un cupo con los referentes institucionales de cada lugar, quienes gestionaron el envío de la encuesta y la recepción de las respuestas. En

El protocolo de la encuesta puede ser solicitado al autor. otros casos la colaboración brindada fue reenviar la encuesta a toda la base de extensionistas del territorio y en otros, se autorizó a realizar un envío a las direcciones de correo electrónico que figuraban en las páginas institucionales.

Se aclara que por tratarse de técnicos que trabajan en zonas rurales de todo el país, éstos no siempre cuentan con acceso fluido a internet. Incluso, en muchos casos no poseen cuentas de correo electrónico propias, sino que usan direcciones genéricas de las agencias locales de las que forman parte. Así, resulta difícil evaluar la tasa de devolución efectiva, teniendo en cuenta que si bien se estima que la encuesta fue recibida por la mayoría de los extensionistas de las instituciones abordadas, las distintas gestiones realizadas en cada territorio y las limitaciones de acceso a internet de los destinatarios hacen este guarismo incierto.

Con respecto a la distribución territorial de las respuestas, se categorizó como región $\mathrm{Cu}$ yo a: Mendoza, San Luis y San Juan, como Noreste (NEA) a: Chaco, Corrientes, Formosa y Misiones; como Noroeste (NOA) a: Catamarca, Jujuy, La Rioja, Salta, Santiago del Estero y Tucumán; como Pampeana a: Buenos Aires, Córdoba, Entre Ríos, La Pampa y Santa Fe; y como Patagonia a: Chubut, Neuquén, Río Negro, Santa Cruz y Tierra del Fuego. Así, teniendo en cuenta 5 casos perdidos, Cuyo contó con 14 respuestas, NEA con 46, NOA con 61, Pampeana con 80 y Patagonia con 13, una distribución consistente con la cantidad de explotaciones agropecuarias presentes en las distintas regiones.

La encuesta utilizada corresponde a una investigación más amplia. Aquí sólo se profundiza en las preguntas correspondientes al estudio que se informa. La encuesta contó con dos partes: La primera incluye preguntas cerradas dedicadas a obtener información sociodemográfica como sexo, edad, institución de pertenencia, provincia donde trabaja, máximo nivel educativo completo (0: ninguno, 1: primario, 2: secundario, 3 : terciario no universitario, 4: universitario, 5 : cursos de postgrado, 6: especialización, 7: maestría, 8: doctorado), título universitario obtenido (nin- 
guno, Ing. Agrónomo, Veterinario, Ciencias Sociales, Otros) y experiencia en el trabajo de extensión. La segunda parte incluye fundamentalmente preguntas abiertas, las cuales abordan los problemas a los que se enfrentan los extensionistas en su práctica, la evaluación de si la Psicología puede contribuir a la solución de alguno de ellos y cuáles podrían ser dichas contribuciones. Buscando evaluar la pertinencia y facilidad de comprensión de las preguntas, se efectuó una prueba piloto con 10 voluntarios utilizando una versión preliminar de la encuesta, sobre la que se hicieron pequeños ajustes.

Para analizar las respuestas se procedió de la siguiente forma: Primero, utilizando el apoyo del software Atlas TI 6.2 y siguiendo los lineamientos de la teoría fundamentada (Glaser \& Strauss, 1967), se construyeron categorías para ordenar y sintetizar las respuestas a las preguntas abiertas. Para esto, los investigadores realizaron una primera lectura de las respuestas y construyeron dos ejes de análisis, uno referido a con quiénes podrían trabajar los psicólogos en el ámbito de la extensión rural y otro relacionado con las áreas o temas respecto de los cuales la Psicología podría contribuir. Dentro del primer eje, se identificaron tres categorías: técnicos, productores y a nivel institucional o supra-institucional. Para el segundo eje se construyó un listado provisorio de categorías referidas a los distintos temas mencionados por los encuestados.

Luego, todas las respuestas fueron categorizadas de acuerdo con el primer eje, como 'trabaja con técnicos', 'con productores' o 'a nivel institucional o supra-institucional' cuando uno o más fragmentos de las preguntas abiertas analizadas hacían referencia a que los psicólogos podían trabajar con cada uno de estos interlocutores. En este proceso, estas categorías no se consideraron excluyentes. Asimismo, cuando el texto de las respuestas resultaba ambiguo con respecto a alguna de las tres categorías, se optaba por excluir esa respuesta, lo que correspondía estadísticamente a un valor perdido. En paralelo, los fragmentos de las respuestas abiertas también fueron categorizados según los componentes del se- gundo eje. En este proceso, se construyeron definiciones de las categorías así como criterios para tomar decisiones ante situaciones dudosas que surgían en torno a la categorización. Así, al finalizar esta fase de la categorización, los investigadores arribaron a un conjunto de categorías definidas de manera clara asociadas a criterios concretos de toma de decisiones en relación a casos dudosos. En base a estas definiciones se revisó nuevamente todo el material y se ajustó la categorización a los parámetros de las definiciones finales obtenidas. Además, para chequear la consistencia de cada categoría, se leyeron todos los fragmentos referidos a cada una de ellas, realizándose pequeños ajustes y unificándose aquellas de límites borrosos o contenidos conceptualmente asociados.

Seguidamente, se incorporaron las variables sociodemográficas al SPSS 17.0, así como la variable dicotómica de la segunda parte de la encuesta. Para hacer lo propio con las categorías surgidas de las preguntas abiertas, se consideró a cada una de ellas como una variable dicotómica que podía estar presente o ausente en una encuesta determinada. Así, se consideró presente a cada uno de los temas o ámbitos de trabajo expresados en las categorías cuando ellas habían sido utilizadas para categorizar la encuesta o alguno de sus fragmentos. En relación al análisis de con quiénes podrían trabajar los psicólogos, se consideraron perdidas aquellas respuestas que resultaban ambiguas o dudosas respecto de las categorías ya definidas para dicho análisis.

Primero se analizaron las frecuencias de las respuestas a la pregunta sobre si la Psicología puede o no ayudar a resolver algunos de los problemas a los cuales se enfrentan los técnicos en el trabajo de extensión. Luego se describen las explicaciones dadas por los encuestados que consideraron que la Psicología no puede contribuir, y después se relaciona esta variable con aquellas de carácter sociodemográfico mencionadas previamente. A continuación, se enumeran las contribuciones que puede realizar la Psicología al trabajo de extensión, mencionadas por más del $5 \%$ de los encuestados, se describen los contenidos de cada una de ellas y se analiza la relación 
de aquellas mencionadas por más del $10 \%$ con distintas variables sociodemográficas.

En cuanto a la selección de estadísticos para el estudio de relaciones entre variables, se utilizó Chi Cuadrado cuando se trata de dos variables nominales, el cual se reemplaza por el coeficiente de contingencia cuando los casilleros con valores esperados menores a 5 superan el $20 \%$ del total. Por su parte, dado que no puede asumirse normalidad para las variables edad, años de experiencia en el trabajo de extensión y nivel educativo (estadístico de Kolmogorov-Smirnov: $p<.001$ en los tres casos), cuando estas sean relacionadas con nominales dicotómicas se utilizará la Prueba $U$ de Mann-Whitney.

\section{RESULTADOS Y DISCUSIÓN}

\section{Potencialidad de LA PSicología PARA CONTRIBUIR A LA EXTENSIÓN RURAL}

Se preguntó a los extensionistas rurales si la Psicología podía ayudar a resolver algunos de los problemas que enfrentan en su práctica. De las 219 encuestas, en 193 casos la respuesta fue afirmativa, en 24 fue negativa y en 2 no hubo respuesta. Los resultados porcentuales se presentan en la Figura 1.

Si bien a continuación se analizarán las contribuciones potenciales de la Psicología mencionadas por los encuestados, también es importante conocer las razones por las cuales esta posibilidad es rechazada por el $11 \%$ de los integrantes de la muestra. De los 24 que respondieron en forma negativa, 22 explicaron sus razones. El argumento más frecuente (10 casos) es que la Psicología no puede contribuir porque los problemas de los productores y del trabajo de extensión son económicos y políticos, no psicológicos. Por ejemplo, "creo que son problemas directamente relacionados a políticas públicas y de Estado para el sector y no encuentro relación con la Psicología". Es de hacer notar que en 6 casos en los que la respuesta fue negativa en términos generales, consideraron que la Psicología podría realizar algunas contribuciones específicas, por ejemplo: "me parece que por ahí la Psicología sí podría ayudar a la extensión; ahora, esto no es ayudar a los pequeños productores en sus problemáticas, sino a los técnicos en la forma en que piensan y planifican la intervención". Luego, en 4 sujetos argumentan que en realidad no se sabe suficiente de la Psicología para contestar afirmativamente a la pregunta: "no soy psicólogo, y no sé si puede servir o no". Finalmente, 7 encuestados mencionan otros argumentos, como que se trata de problemas culturales y no psicológicos, o que no se cree que la Psicología tenga capacidad para cambiar la mentalidad de los productores como se desearía.

En la Tabla 1 se analiza la relación entre las variables sociodemográficas seleccionadas y la creencia de que si la Psicología posee o no potencialidad para contribuir al ámbito de la extensión rural.

Se observa que únicamente el nivel educativo se relaciona con la creencia de que la Psicología puede contribuir al trabajo de extensión. En concreto, quienes consideran que la Psicología puede contribuir, tienen una media de nivel educativo igual a 4.61, frente a 4.09 de quienes no lo consideran así.

En primer lugar, se observa que un alto porcentaje de sujetos considera que la Psicología podría contribuir al trabajo de extensión. Esto contrasta con dos hechos: El primero es que en la muestra no hay profesional alguno de la Psicología, lo que sería esperable si la mayoría de quienes trabajan en extensión lo consideran recomendable. El segundo se refiere a la escasez mencionada de contribuciones de la Psicología, tanto a nivel teórico como profesional, a los procesos de desarrollo rural, particularmente en lo que hace a la mejora de las estrategias de trabajo e intervención con productores (Landini, Benítez $\&$ Murtagh, 2011). De esto surge que el ámbito de la extensión y el desarrollo rural constituyen un área que posee demanda social y de vacancia para la Psicología.

En cuanto a las explicaciones dadas por quienes consideraron que la Psicología no podía contribuir al trabajo de extensión, resalta cuantitativamente aquella que sostiene que los problemas vinculados con la exten- 
sión y el desarrollo son de índole económica y política y no psicológica, argumento que se esperaba encontrar con mayor frecuencia. En contrapartida, son llamativos los casos en los que el encuestado manifiesta que la Psicología no puede contribuir (al responder a la variable dicotómica propuesta por el cuestionario), pero al argumentar su respuesta, hace referencia a contribuciones potenciales. $\mathrm{Si}$ a esto se suman los que señalaron que no saben suficiente de la Psicología como para responder afirmativamente, se entrevé que este rechazo puede estar más relacionado con el desconocimiento de la Psicología como disciplina y de la dimensión psicológica y psicosocial de ciertos procesos interpersonales y colectivos, que con una opinión formada conociendo en qué cuestiones y de qué manera esa contribución podría concretarse. De esto se sigue el interés de poder clarificar y dar a conocer este rol potencial para obtener opiniones más formadas.

Finalmente, la única variable sociodemográfica analizada que se relaciona con un mayor porcentaje de respuestas afirmativas es el mayor nivel educativo. Posiblemente, esto se vincule con un mayor conocimiento sobre el campo de las ciencias sociales como área de interés, estudio de postgrado característico de quienes trabajan en extensión (Landini, 2012a).

\section{Contribuciones de la Psicología al tRabajo de} EXTENSIÓN RURAL

En la Tabla 2 se presentan las áreas temáticas o las modalidades propuestas por más del 5\% de los 193 encuestados que respondieron afirmativamente a la pregunta de si la Psicología puede contribuir al trabajo de extensión rural. Ellas son:

1.- Conocimiento experto en el área de gestión y manejo de grupos: se argumenta que el psicólogo posee capacidad para apoyar, formar, asesorar o gestionar procesos grupales, por lo que puede contribuir en el área de construcción de asociaciones, superación del individualismo y la desconfianza, manejo de diferencias personales, construcción de liderazgos y manejo de grupos. Como sintetiza un encuestado, "la Psicología Social puede aportar en la conformación y organización de grupos de productores, identificación de procesos de autogestión, consolidación de grupos y redes de trabajo, acompañamiento en la formación de líderes y fortalecimiento de [...] los grupos".

2.- Capacitar, asesorar o proveer de herramientas a extensionistas rurales: los encuestados sostienen que la Psicología puede proveer tanto conocimientos como herramientas o técnicas específicas para enfrentar y resolver diversos problemas. En la mayoría de los casos, esto se argumenta genéricamente, como cuando se dice que los psicólgos pueden actuar como "capacitadores de los extensionistas" o que pueden "dar herramientas para tener sensibilidad y detectar problemas o dificultades". No obstante, también existen casos en los cuales esto se especifica, señalando áreas en las cuales el asesoramiento o la capacitación son esperados, como cuando se afirma que pueden participar "capacitando agentes de desarrollo en técnica operativa de grupo".

3.- Formar parte de equipos interdisciplinarios de extensión rural: se menciona que los profesionales de la Psicología podrían contribuir integrándose en los equipos territoriales por medio de los cuales se encuentran organizadas las tareas, destacándose en muchos casos la necesidad de la interdisciplina, lo que incluye, pero va más allá, de la presencia de la Psicología. En definitiva, se trata de que "la Psicología puede ser incorporada en los equipos de trabajo de extensión", es decir, "que técnicos especializados en Psicología formen parte de equipos de trabajo multidisciplinarios, y que entre todos podamos abordar los problemas en forma integral".

4.- Los psicólogos pueden dar capacitación o gestionar procesos grupales con productores: es decir, que ellos pueden llevar a cabo diferentes actividades en interacción directa con los productores, lo que incluye 
tanto dar capacitaciones como coordinar distintos tipos de procesos grupales, destacándose aquellos de naturaleza participativa. Por ejemplo, se argumenta que los psicólogos pueden "ayudar en la dinámica grupal, en la resolución de conflictos, en colaborar para que las personas puedan manifestar sus necesidades y problemas", resultando pertinente el uso de "metodologías de la dinámica grupal con aplicación de técnicas participativas".

5.- Ayudar a comprender al productor: refiere a la experiencia de los extensionistas de que diferentes acciones de los beneficiarios no tienen sentido desde su propio punto de vista, como puede ser la falta de adopción de tecnologías consideradas más eficientes, experiencia que ha sido destacada por diferentes investigadores (e.g. Cáceres, 2003; Cittadini et al., 2001; Cittadini \& Pérez, 1996; Landini, 2011b). Como señala un encuestado, la Psicología podría "ayudarnos a los extensionistas a tratar de entender mejor la psicología del productor".

6.- Dar apoyo pedagógico o comunicacional: destaca la posibilidad de la Psicología de capacitar o asesorar en la mejora de la comunicación y en el abordaje pedagógico de los productores. La siguiente cita constituye una buena síntesis de esta categoría: "[puede aportar] didácticas para diseñar [...] sistemas de aprendizaje de la mejor manera, mejorar la comunicación con ellos, y entre los profesionales que trabajamos en este ámbito".

7.- Participar en la planificación de proyectos o procesos: se argumenta que los psicólogos pueden trabajar en el ámbito de la extensión actuando en procesos de planificación tanto con los productores (diagnóstico, programación, ejecución y evaluación) como en el interior de los equipos de extensión. Como explica un entrevistado, "[la psicología] es una disciplina de suma importancia en el ciclo de los proyectos, principalmente en el diagnóstico y en etapas de evaluación".

8.- Resolver o gestionar conflictos que también se incluye mayormente en la catego- ría (1), refiere a la capacidad de la Psicología para evitar o ayudar a superar conflictos interpersonales que pueden darse a distintos niveles, fundamentalmente en el contexto de grupos de productores pero también a nivel institucional, familiar o directamente interpersonal. Como dice una encuestada, un psicólogo "podría ser de gran ayuda al momento en que se presentan los conflictos, tanto dentro como fuera del grupo, muchas veces los técnicos no sabemos cómo manejarlos".

9.- La Psicología podría "generar compromiso y/o participación de parte de los productores" teniendo en cuenta lo que los extensionistas describen como 'actitudes pasivas' de los productores (Landini, 2012b), en distinto tipo de iniciativas, ayudando a "entender cómo podemos intervenir o de qué forma, para ayudarlos a participar o involucrarse más" con el fin de lograr "un verdadero compromiso por parte de las poblaciones objetivo de las intervenciones sociales".

10.- Aumentar o fortalecer la autoestima de los productores en un contexto en el que se argumenta que "estos sectores [los pequeños productores] tienen tendencia a menospreciarse". Se sostiene que "la psicología podría estar trabajando en lo que es la autoestima, la valoración personal".

11.- Brindar apoyo a la adopción de tecnologías y al cambio de mentalidad de los productores: esto implica por un lado, proveer estrategias para que los productores adopten las propuestas presentadas por los técnicos ("facilitar metodología que facilite la apropiación de las tecnologías") y por otro, apoyar un cambio en las actitudes y el modo de pensar de los productores (contribuir "con metodologías [...] para cambiar algunas actitudes y permitir el desarrollo").

12.- Dar apoyo emocional o terapéutico o trabajar con problemas de salud mental, ya que "la ayuda psicológica siempre es útil" y "la gente campesina también se deprime, tiene angustias y temores". 
13.- Trabajar en el área de género, juventud y niñez, brindando herramientas para abordar las problemáticas específicas y las necesidades propias de estos grupos, dando "apoyo al trabajo con jóvenes, mujeres y niños para el desarrollo, cómo abordar en un proyecto de desarrollo".

14.- Intervenir a nivel del equipo técnico o institucional, lo que en general refiere a trabajar con los equipos de extensionistas o con las organizaciones de extensión mejorando los vínculos y facilitando las dinámicas de trabajo. Esto incluye tanto "mejorar la gestión de conducción institucional" como "ayudar a mejorar la comunicación y un buen funcionamiento del equipo técnico".

15.- Mejorar la relación entre técnicos y productores: se refiere a "apoyar a una mejor interacción entre el extensionista y el pequeño productor", brindando herramientas y sugiriendo estrategias para una mejor comunicación y para generar relaciones empáticas.

16.- Transformar o modificar la forma de pensar de los extensionistas en distintas áreas, por ejemplo "atenuar la soberbia profesional", "inculcar los beneficios de trabajar en equipo" o "ayudar para mejorar la forma de 'intervenir' de los técnicos, permitiendo un trabajo menos protector".

17.- Aportar al desarrollo de procesos reflexivos, lo que se expresa en "asesorar en estrategias para poder repensar sobre conceptos preestablecidos" o "facilitar la reflexión con equipos de terreno sobre problemas concretos".

18.- Ayudar a comprender la lógica de los actores locales, aportando "herramientas a la intervención en los procesos de lectura [...] de acciones de los grupos, organizaciones e instituciones".

19.- Investigar en el ámbito de la extensión rural o los problemas rurales. Para un análisis consistente de las áreas de contribu- ción potencial de la Psicología a la extensión rural, resulta fundamental tener en cuenta: por un lado, las diferencias porcentuales en la aparición en las encuestas, ya que algunas cuestiones poseen una presencia muy fuerte, mientras que otras sólo son mencionadas esporádicamente. Por el otro, el carácter espontáneo de la mención de las distintas áreas (producto de la estructura de la pregunta abierta con la que se trabajó el tema), lo que podría incrementar los porcentajes sustancialmente en caso de usarse un método diferente, como sería el de la presentación de un listado de opciones.

A partir de las 11 primeras propuestas de contribución de la Psicología al trabajo de extensión rural, señaladas por más del $10 \%$ de los encuestados, puede concluirse que aquellos que valoran tales contribuciones en el ámbito de la extensión rural, sugieren la incorporación de psicólogos a sus equipos de trabajo (3), teniendo como actividades genéricas capacitar, asesorar o proveer herramientas a los extensionistas rurales (2) y participar en espacios de planificación de proyectos (7), contribuyendo en diferentes áreas como ser procesos asociativos y manejo de grupos (1), comprensión de la idiosincrasia y la racionalidad cultural de los productores (5), estrategias pedagógicas y de comunicación (6), resolución de conflictos (8) y solución de problemas vinculados con la falta de participación y compromiso de los productores con los proyectos (9) y con la falta de adopción de tecnologías (11). También tendrían un rol específico en el trabajo directo con productores, dando capacitación y gestionando procesos grupales participativos (4), ocupándose entre otros temas del aumento y fortalecimiento de la autoestima de los productores (10).

Así, se delinea un rol específico para el trabajo de los psicólogos en el ámbito de la extensión rural que en gran medida se corresponde con las capacidades de los profesionales formados en las universidades en el ámbito de la Psicología Social, institucional o comunitaria. En concreto, existen interesantes desarrollos de la Psicología en el área de la intervención comunitaria y la planifi- 
cación participativa (Lapalma, 2012; Sánchez Vidal, 1991), los procesos grupales y las conductas asociativas (Kelley \& Stahelski, 1970; Landini, 2007b; Páez \& Campos, 2004; Rabbie, 1995), las racionalidades de los actores sociales (Landini, 2011b; Lapalma, 2001), los procesos de comunicación (Wainstein, 2002, Watzlawick, Beavin \& Jackson, 1971) y los posicionamientos pasivos de los sujetos, estudiados por la Psicología en términos de indefensión aprendida (Hunziker, 2005; Seligman, 1989; Yela Bernabé, Gómez \& Salgado Ruiz, 2001), locus de control (Hansemark, 2003; Oliver, Jose \& Brough, 2006) y fatalismo (Blanco \& Díaz, 2007; Martín-Baró, 1987). En consecuencia, se hace evidente que las expectativas de los extensionistas están en línea con las capacidades de la Psicología.

\section{VARIABLES ASOCIADAS A LAS CONTRIBUCIONES DE LA PSICOLOGÍA AL TRABAJO DE EXTENSIÓN RURAL}

Con el fin de analizar el perfil de los extensionistas que proponen distintas áreas de trabajo para los psicólogos en el ámbito de la extensión rural, se analizó la relación entre las propuestas y otras variables de interés, como se observa en la Tabla 3.

Analizando las relaciones significativas identificadas entre las variables, se observa que las mujeres mencionan más frecuentemente la potencialidad de la Psicología para trabajar en el área de asociativismo y manejo de grupos (1) $(69 \%$ de las mujeres frente al $47 \%$ de los hombres), para capacitar productores o gestionar grupos (4) $(50 \%$ frente al $34 \%$ ) y para ayudar a comprender a los productores (5) (37\% frente al 21\%). Estos resultados podrían deberse a la existencia de perfiles de formación diferentes entre hombres y mujeres o a que ciertas instituciones, más orientadas al trabajo con grupos o asociaciones como es el caso de la Subsecretaría de Agricultura Familiar, cuentan con más mujeres. No obstante, ninguna de las áreas de contribución que poseen una relación significativa con sexo se asocia a institución o a título académico, por lo que estas explicacio- nes no encuentran soporte en los datos. Así, parece razonable argumentar una sensibilidad particular de las mujeres en relación a las áreas de intervención mencionadas previamente sin que surjan explicaciones alternativas relacionadas con ciertas variables que pudieran estar asociadas al sexo.

Por su parte, también se observa que la media de edad de quienes apoyan la contribución (1) es menor (39.86 años) que la de quienes no lo hacen (44.25 años), sucediendo lo mismo en el caso de la contribución (8), referido a la potencialidad de los psicólogos para resolver y gestionar conflictos (37.63 años es la media de quienes mencionan esta área, mientras que la de quienes no lo hacen es de 42.61 años).

Por su parte, no se observan asociaciones significativas entre las áreas de contribución analizadas y la institución de pertenencia, la región del país en la que trabajan los encuestados o la experiencia en el trabajo de extensión. Con respecto a la institución de pertenencia esto resulta llamativo, ya que podría esperarse, al menos en el caso de las propuestas relacionadas con el trabajo grupal, mayor incidencia en el caso de la SAF y menor en el ProHuerta, dada la mayor importancia que adquiere el trabajo con grupos de productores en la primera con respecto al segundo. También se observa que quienes destacan el interés de que los psicólogos formen parte de equipos interdisciplinarios de extensión rural (3) y a la vez participen en trabajos vinculados con la planificación (7), tienen en promedio un mayor nivel educativo que quienes no lo hacen (4.95 contra 4.57 y 5.00 contra 4.43, respectivamente).

En relación al título académico de los extensionistas, se observan asociaciones significativas en las áreas referidas a formar parte de equipos interdisciplinarios de extensión rural (3) y a brindar apoyo a la adopción de tecnologías y al cambio de mentalidad de los productores (11). En el caso de la formación de equipos interdisciplinarios, tema mencionado por un $40 \%$ de los encuestados que también respondieron a la pregunta de su formación académica, se observa un porcentaje mayor en el caso de los veterinarios 
$(69.23 \%)$, menor en quienes no poseen título universitario (15\%) o tienen títulos categorizados como 'otros' (29.41\%), encontrándose cerca de la media los ingenieros agrónomos (43.24\%) y los profesionales de las ciencias sociales $(43.75 \%)$. En cuanto al apoyo a la adopción de tecnologías, mencionado en el $9.44 \%$ de los casos donde se indicó formación académica, se observa un porcentaje claramente mayor en quienes provienen de 'otras disciplinas' $(29.41 \%)$, uno menor en los veterinarios $(0 \%)$ y en los cientistas sociales $(6.25 \%)$, mientras que porcentajes más cercanos al promedio en los ingenieros agrónomos $(8.11 \%)$ y en quienes no poseen formación universitaria $(8.70 \%)$. Resulta interesante mencionar el caso de los veterinarios, ya que destacan más la importancia de la interdisciplina, a la vez que proponen una Psicología no orientada a la transferencia de tecnologías o al cambio de mentalidad de los productores, lo que podría sugerir un perfil propio, relacionado con una mirada más dialógica y comunicativa de la extensión rural frente a otras profesiones, algo que no ha sido sugerido anteriormente.

\section{CON QUIÉNES PUEDEN TRABAJAR LOS PSICÓLOGOS}

A los encuestados que afirmaban que la Psicología podía contribuir al trabajo de extensión rural, se les preguntó con quiénes podrían trabajar los psicólogos en ese ámbito. Las respuestas se agruparon en tres grandes áreas: el trabajo con productores, con técnicos (área que incluyó el trabajo con equipos de extensión) y a nivel institucional o suprainstitucional. Además, para cada categoría, se excluyeron aquellas respuestas que resultaban ambiguas debido a que se referían a una o más de estas tres áreas, considerando perdidos esos casos en relación a la variable que presentaba dudas. El trabajo con técnicos fue mencionado en 135 casos, el trabajo con productores en 117 y la intervención a nivel institucional o supra-institucional en 19 oportunidades, lo que constituye un $80.80 \%$, un $69.60 \%$ y un $11.00 \%$ de los casos válidos, respectivamente.
Se observa que los encuestados enfatizan en la capacidad de los profesionales de la Psicología para trabajar con técnicos y con productores, en detrimento del trabajo a nivel institucional, evidenciándose un porcentaje más alto en el caso del trabajo con técnicos. El caso prototípico del trabajo con técnicos es la participación en equipos de extensión rural o la capacitación y asesoramiento a extensionistas. Por su parte, la idea de dar capacitación a productores o coordinar con ellos procesos grupales hace referencia clara a la idea de trabajar con productores. En cuanto al trabajo en el ámbito institucional o suprainstitucional, la incidencia es claramente más baja y hace referencia a diferentes alternativas, las que incluyen la facilitación de la articulación entre diversas instituciones, la mejora del clima institucional y la participación en el diseño de políticas o programas de desarrollo rural.

\section{Conclusiones}

Si bien en múltiples trabajos previos se ha argumentado en términos generales la potencialidad de la Psicología para trabajar en el ámbito de la extensión y el desarrollo rural (Barilari, Landini, Logiovine \& Rotman, 2011), en el presente artículo se ha brindado evidencia empírica de que un porcentaje significativo de los extensionistas rurales que trabajan en las principales insti-tuciones públicas argentinas comparten esta posición. Se concluye que existe un amplio interés y una demanda potencial en este ámbito, lo que exige profesionales de la Psicología formados en el área. De la misma manera, los resultados del estudio realizado también han permitido construir un perfil de psicólogo requerido. En concreto, se trata de un profesional con formación social que trabaje tanto con los extensionistas brindando capacitaciones, asesoramiento y siendo parte de equipos territoriales interdisciplinarios, como trabajando directamente con productores, particularmente en la gestión de procesos grupales. Como ámbitos temáticos, se destaca la potencialidad de la Psicología en el área del 
asociativismo y el trabajo grupal, pero también se señala su competencia en torno a la comprensión de la idiosincrasia de los productores, el fortalecimiento de su autoestima, los procesos de comunicación interpersonal, la influencia para el cambio de prácticas y actitudes y la implicación en procesos colectivos.

Ciertamente, resulta importante no omitir las limitaciones de este trabajo. Tratándose de una encuesta voluntaria donde hubo un nivel de respuesta bajo, es posible que sólo hayan optado por responder aquellas personas interesadas en el rol de la Psicología. De cualquier manera, hay otras razones que pueden explicar el bajo nivel de respuesta, particularmente problemas para acceder a internet y tendencia a trabajar en el campo en lugar de dedicarse a actividades de tipo burocráticas, dentro de las cuales puede encuadrarse responder a esta encuesta. En cualquier caso, la duda permanece. En las oportunidades en las cuales se ha trabajado capacitando o asesorando extensionistas (Landini, en prensa;
Landini, Bianqui \& Russo, 2012), sin que los destinatarios pudieran optar por otros profesionales, la respuesta ha sido muy positiva. De todas maneras, esto no quita la necesidad de realizar investigaciones posteriores que utilicen muestras probabilísticas y no incidentales, como es este caso.

Para finalizar, resta señalar que el trabajo que se informa constituye una apertura orientada a visibilizar un espacio potencial de investigación e intervención de la Psicología, hasta la fecha escasamente considerado. Y esto, no pensando en generar nuevos espacios de inserción profesional en tanto un bien para los psicólogos, sino desde el compromiso de quien piensa que la Psicología puede y debe comprometerse con el cambio social (Montero, 2004). En este caso, convirtiéndose en un instrumento para el desarrollo rural de los sectores más desfavorecidos del campo. Estos resultados constituyen un mapa que puede orientar acciones de intervención e investigación. Resta, sin embargo, asumir el compromiso y recorrer el camino. 
FIGURA 1

OPINIÓN DE LOS EXTENSIONISTAS SOBRE SI LA PSICOLOGÍA PUEDE AYUDAR A RESOLVER ALGUNOS DE LOS PROBLEMAS QUE SE PRESENTAN EN EL TRABAJO DE EXTENSIÓN RURAL

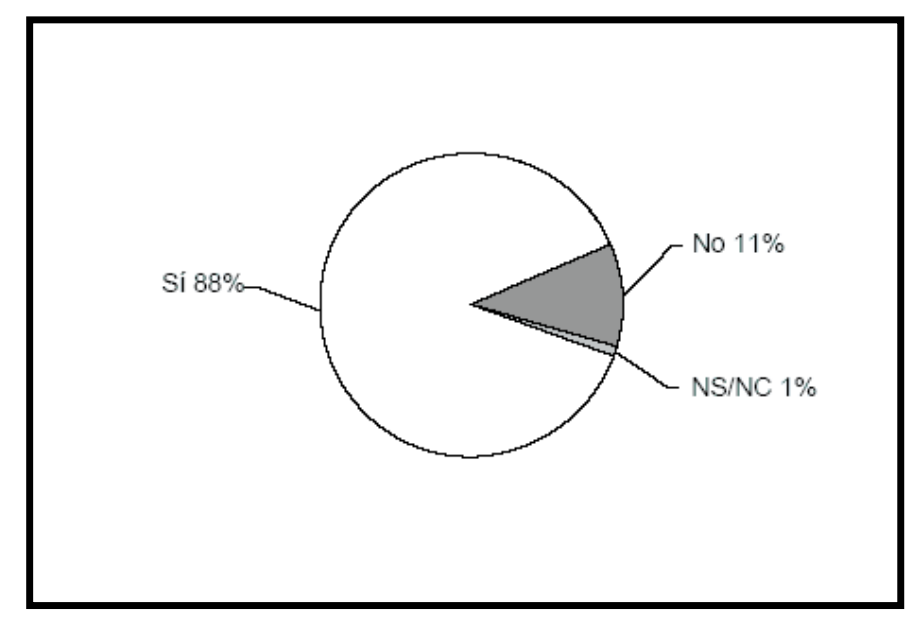

TABLA 1

CAPACIDAd DE LA PSICOlogía PARA CONTRIBUIR AL TRABAJO DE EXTENSIÓN Y SU RELACIÓN CON DIVERSAS VARIABLES

\begin{tabular}{|c|c|c|c|c|c|c|}
\hline $\begin{array}{c}\text { Sexo } \\
\chi^{2}\end{array}$ & $\begin{array}{c}\text { Edad } \\
\text { MW: } z\end{array}$ & $\begin{array}{c}\text { Institución } \\
\chi^{2}\end{array}$ & $\begin{array}{c}\text { Región } \\
\text { Coef. }\end{array}$ & $\begin{array}{c}\text { Nivel educativo } \\
\text { MW: } z\end{array}$ & $\begin{array}{c}\text { Título } \\
\text { Coef. }\end{array}$ & $\begin{array}{c}\text { Experiencia } \\
\text { MW: } z\end{array}$ \\
\hline $1.19(1)$ & -.83 & $2.46(2)$ & .11 & $-2.29^{*}$ & .19 & -.10 \\
\hline
\end{tabular}

Nota

Coef.: Coeficiente de contingencia

MW: $U$ de Mann-Whitney

${ }^{*} p<.05$ 
TABLA 2

CONTRIBUCIONES de LA PSICOLOGÍA AL TRABAJO DE EXTENSIÓN RURAL

\begin{tabular}{|l|cc|}
\hline \multicolumn{1}{|c|}{ Contribuciones } & Frecuencia & Porcentaje \\
\hline & & \\
\hline 1.- Conocimiento experto en el área de gestión y manejo de grupos. & 106 & 54.9 \\
2.- Capacitar, asesorar o proveer de herramientas a extensionistas rurales. & 83 & 43.0 \\
3.- Formar parte de equipos de extensión rural interdisciplinarios. & 78 & 40.4 \\
4.- Dar capacitación o gestionar procesos grupales con productores. & 77 & 39.9 \\
5.- Ayudar a comprender al productor. & 52 & 26.9 \\
6.- Dar apoyo pedagógico o comunicacional. & 34 & 17.6 \\
7.- Participar en la planificación de proyectos o procesos (diagnóstico, progra- & & 16.1 \\
mación, evaluación). & 31 & 15.5 \\
8.- Resolver o gestionar conflictos. & 30 & 14.5 \\
9.- Generar compromiso y/o participación de parte de los productores. & 28 \\
10.- Aumentar o fortalecer la autoestima de los productores. & 24 & 10.9 \\
11.- Brindar apoyo a la adopción de tecnologías y al cambio de mentalidad de & & \\
los productores. & 21 & 9.8 \\
12.- Dar apoyo emocional o terapéutico o trabajar con problemas de salud & & 9.3 \\
mental. & 19 & 8.8 \\
13.- Trabajar en el área de género, juventud y niñez. & 18 & 8.3 \\
14.- Intervenir a nivel del equipo técnico o institucional. & 17 & 7.3 \\
15-. Mejorar la relación entre técnicos y productores. & 16 & 5.7 \\
16.- - Transformar o modificar la forma de pensar de los extensionistas. & 14 & 5.2 \\
17.- Aportar al desarrollo de procesos reflexivos. & 11 & 5.2 \\
18.- Ayudar a comprender la lógica de los actores locales. & 10 & \\
19.- Investigar en el ámbito de la extensión rural o los problemas rurales. & 10 & \\
& & \\
\hline
\end{tabular}


TABLA 3

CONTRIBUCIONES de LA PSICOLOGía Y SU RELACIÓN CON DIVERSAS VARIABLES

\begin{tabular}{|c|c|c|c|c|c|c|c|}
\hline $\begin{array}{c}\text { Contribu- } \\
\text { ciones }\end{array}$ & $\begin{array}{c}\text { Sexo } \\
\chi^{2}\end{array}$ & $\begin{array}{c}\text { Edad } \\
\text { MW: } z\end{array}$ & $\begin{array}{c}\text { Institución } \\
\chi^{2}\end{array}$ & $\begin{array}{c}\text { Región } \\
\chi^{2} / \text { Coef. }\end{array}$ & $\begin{array}{c}\text { N. educativo } \\
\text { MW: } z\end{array}$ & $\begin{array}{c}\text { Título } \\
\chi^{2} / \text { Coef. }\end{array}$ & $\begin{array}{c}\text { Experiencia } \\
\text { MW: } z\end{array}$ \\
\hline 1 & $8.26(1)^{* *}$ & $-2.95^{* *}$ & $1.26(2)$ & $6.30(4)$ & -1.85 & $3.99(4)$ & -1.31 \\
2 & $.11(1)$ & -.36 & $1.37(2)$ & $6.78(4)$ & -.70 & $3.09(4)$ & -.76 \\
3 & $.01(1)$ & -.02 & $4.60(2)$ & $4.61(4)$ & $-2.98^{* *}$ & $12.97(4)^{*}$ & -1.43 \\
4 & $4.68(1)^{*}$ & -.96 & $.57(2)$ & $3.19(4)$ & -.93 & $3.93(4)$ & -.10 \\
5 & $5.80(1)^{*}$ & -.85 & $4.00(2)$ & $4.61(4)$ & -.65 & .14 & -.84 \\
6 & $.27(1)$ & -1.39 & $.13(2)$ & $3.41(4)$ & -.11 & .06 & -.82 \\
7 & $.51(1)$ & -.28 & $5.98(2)$ & $4.45(4)$ & $-2.23^{*}$ & .15 & -.83 \\
8 & $2.90(1)$ & $-2.68^{* *}$ & $.19(2)$ & $4.32(4)$ & -1.28 & .09 & -1.51 \\
9 & $.01(1)$ & -.15 & $1.04(2)$ & $3.10(4)$ & -.82 & .08 & -.78 \\
10 & $.34(1)$ & -.79 & $3.15(2)$ & .09 & -.10 & .07 & -.63 \\
11 & $.60(1)$ & -.90 & $1.67(2)$ & .19 & -1.15 & $.23^{*}$ & -1.32 \\
\hline
\end{tabular}

Nota:

El número de las contribuciones corresponde a las que figuran en la Tabla 2.

$\chi^{2} /$ Coef.: los valores con grados de libertad entre paréntesis corresponden a $\chi^{2}$, el resto al coeficiente de contingencia. Coef.: coeficiente de contingencia

MW: $U$ de Mann-Whitney

${ }^{* *} p<.01$

${ }^{*} p<.05$ 


\section{Referencias bibliográficas}

Albuquerque, R. (1995). Estratégia de desenvolvimento e combate à pobreza [A strategy for development and for fighting against poverty]. Estudos Avançados, 24, 75-116. doi:10. 1590/S010340141995000200004.

Alemany, C. \& Sevilla Guzmán, E. (2007). ¿Vuelve la extensión rural? Reflexiones y propuestas agroecológicas vinculadas con el retorno y fortalecimiento de la extensión rural en América Latina [Is rural extension returning? Reflections and agroecological proposals related to the returning and strengthening of rural extension in Latin America]. Realidad Económica, 227, 52-74.

Barilari, Z., Landini, F., Logiovine, S. \& Rotman, J. (2011). La labor del profesional de la psicología en los proyectos de desarrollo rural orientados a pequeños productores agropecuarios [Psychologists working in rural development projects aimed at small farmers]. Revista Argentina de Psicología, 50, 105-115.

Blanco, A. \& Díaz, D. (2007). El rostro bifronte del fatalismo: Fatalismo colectivista y fatalismo individualista [The two-faced face of fatalism. Collectivist fatalism and individualistic fatalism]. Psicothema, 19(4), 552-558.

Cáceres, D. (2003). El campesinado contemporáneo. En R. Thornton \& G. Cimadevilla (Eds.), La extensión rural en debate. Concepciones, retrospectivas, cambios y estrategias para el Mercosur [Rural extension at debate. Approaches, retrospectives, changes and strategies for the Mercosur] (pp. 173-197). Buenos Aires: Ediciones INTA.

Carballo, C. (2002). Extensión y transferencia de tecnología en el sector agrario argentino [Extension and transfer of technology in Argentine agro]. Buenos Aires: Facultad de Agronomía, Universidad de Buenos Aires.

Cittadini, R., Burges, J., Hamdan, V., Natizon, P., Pérez, R. \& Dedieu, B. (2001). Diversidad de sistemas ganaderos y su articulación con el sistema familiar [Diversity of husbandry systems and its articulation with the family system]. Revista Argentina de Producción Animal, 21(2), 119-135.

Cittadini, R. \& Pérez, R. (1996). La importancia de comenzar entendiendo por qué el productor hace lo que hace. El caso del maíz para forraje [The importance of understanding why farmers do what they do. The case of corn for forage]. Visión Rural, 18, 36-39.

De Schutter, A. (1982). Extensión y capacitación rurales [Rural extension and training]. México: Trillas.

Di Pietro, L. (2001). Hacia un desarrollo integrador y equitativo: Una introducción al desarro1lo local. En D. Burin \& A. Heras (Eds.), Desarrollo local. Una respuesta a escala humana a la globalización [Local development. A human-scale response to globalization], (pp. 11-50). Buenos Aires: CICCUS - La Crujía.

Freire, P. (1973). ¿Extensión o comunicación? La concientización en el medio rural [Extension or communication? Awareness in rural areas]. Buenos Aires: Siglo XXI.

Glaser, B. \& Strauss, A. (1967). The discovery of grounded theory. Strategies for qualitative research. Chicago: Aldine.

Hansemark, O. (2003). Need for achivement, locus of control and the prediction of business start-ups: A longitudinal study. Journal of Economic Psychology, 24, 301-319. doi: 10.1 016/S0167-4870(02)00188-5.

Herrera Tapia, F. (2006). Innovaciones tecnológicas en la agricultura empresarial mexicana. Una aproximación teórica [Technological innovation in Mexican agricultural enterprises. A theoretical approximation]. Gaceta Laboral, 12(1), 91-117. doi:10.1157/13084134.

Hunziker, M. (2005). O desamparo aprendido revisitado: Estudos com animais [Learned helplessness revisited: Animal studies]. Psico- 
logia: Teoría e Pesquisa, 21(2), 131-139. doi:10.1590/S0102-37722005000200002.

Kelley, H. \& Stahelski, A. (1970). Social interaction basis of cooperators' and competitors' beliefs about others. Journal of Personality and Social Psychology, 16(1), 66-91. doi:10. 1037/h0029849.

Klerkx, L., Aarts, N. \& Leeuwis, C. (2010). Adaptive management in agricultural innovation systems: The interactions between innovation networks and their environment. Agricultural Systems, 103, 390-400. doi:10. 1016/j.agsy.2010.03.012

Landini, F. (2007a). Teorías del desarrollo y del subdesarrollo: ¿Sólo una incumbencia de la ciencia económica? Algunos aportes desde la psicología comunitaria. En E. Saforcada, N. Cervone, J. Barriera, A. Lapalma \& M. De Lelis (Comps.), Aportes de la psicología comunitaria a problemáticas de la actualidad latinoamericana [Contributions from community psychology to current Latin American problems] (pp. 273-291). Buenos Aires: Facultad de Psicología, UBA y JVE Ediciones.

Landini, F. (2007b). Prácticas cooperativas en campesinos formoseños. Problemas y alternativas [Cooperative practices in peasants from Formosa: Problems and possible solutions]. Revista de la Facultad de Agronomía, 27(2), 173-186.

Landini, F. (2010). Ingenieros extensionistas desde la mirada de los pequeños productores. Representaciones, expectativas y realidades [Extension engineers from the perspective of small producers. Representations, expectations and realities]. Mundo Agrario, 20 [Versión electrónica]. Recuperado el 1 de noviembre de 2012 de http://www.mundoagrario. unlp.edu.ar/numeros/no-20-1er-sem2010/inge nieros-extensionistas-formosenosdesde-la-mirada-de-los-pequenos-productores-representaciones-expectativas-y-realidades.
Landini, F. (2011a). Income and use of money in the peasant economy. Contributions to rural development psychology from a case study. Journal of Alternative Perspectives in the Social Sciences, 3(3), 674-703.

Landini, F. (2011b). Racionalidad económica campesina [Peasant economic rationale]. Mundo Agrario, 23 [versión electrónica]. Recuperado el 1 de noviembre de 2012 de http:// www.mundoagrario.unlp.edu.ar/numeros/no23-2do-sem-2011/racionalidad-economicacampesina.

Landini, F. (2012a). Perfil de los extensionistas rurales argentinos del sistema público [Profile of the Argentine rural extensionists working in the public system]. Manuscrito enviado para publicación.

Landini, F. (2012b). Pasividad, dinamismo y percepción de control en la vida campesina [Passivity, dynamism and perception of control in peasants' life]. Investigaciones en Psicología, 17(1), 59-79.

Landini, F. (en prensa). Problemas en la extensión rural paraguaya: Modelos de extensión en la encrucijada [Problems in Paraguayan rural extension: Extension models in the crossroads]. Cuadernos de Desarrollo Rural.

Landini, F., Benítez, M. \& Murtagh, S. (2010). Revisión de los trabajos realizados por la psicología sobre pequeños productores agropecuarios [Review of psychological papers on small farmers]. Anuario de Investigaciones de la Facultad de Psicología, 17, 221-229.

Landini, F. \& Bianqui, V. (2012). Can psychology contribute to rural extension? Journal of Alternative Perspectives in the Social Sciences, 4(2), 485-492. doi:10.1002/ casp. 2105.

Landini, F., Bianqui, V. \& Russo, M. (2012). Evaluación de un proceso de capacitación para extensionistas rurales implementado en Paraguay [Evaluation of a training process for rural extensionists implemented in Paraguay] Manuscrito enviado para publicación. 
Landini, F., Long, N., Leeuwis, C. \& Murtagh, S. (2012). Theoretical guidelines for a Psychology of Rural Development. Manuscrito enviado para publicación.

Landini, F., Murtagh, S. \& Lacanna, M. (2009). Aportes y reflexiones desde la psicología al trabajo de extensión con pequeños productores [Contributions and reflections from psychology to rural extension with small farmers]. Formosa, Argentina: Ediciones INTA.

Lapalma, A. (2001). El escenario de la intervención comunitaria [The scenary of community intervention]. Revista de Psicología de la Universidad de Chile, 10(2), 61-70.

Lapalma, A. (2012). Psicología comunitaria. Niveles múltiples en los procesos de intervención comunitaria. En A. Zambrano \& H. Berroeta (Comps.), Teoría y práctica de la acción comunitaria [Theory and practice of community action] (pp. 73-93). Santiago, Chile: RIL Editores.

Leeuwis, C. (2004). Communication for rural innovation. Rethinking agricultural extension. Oxford y Wageningen: Blackwell. doi:10.10 02/9780470995235.

Leeuwis, C. \& Aarts, N. (2011). Rethinking communication in innovation processes: Creating space for change in complex systems. Journal of Agricultural Education and Extension, 17(1), 21-36. doi:10.1080/ 1389224X.2011. 536344.

Leeuwis, C. \& Pyburn, R. (2002). Wheelbarrows full of frogs. Social learning in rural resource management. Assen, Holanda: Van Gorcum.

Machado, J., Hegedüs, P. \& Silveira, L. (2006). Estilos de relacionamento entre extensionistas e produtores: desde uma concepçao bancária até o 'empowerment' [Styles of relation- ship between extension agents and farmers: From the "bankarian conception" to the empowerment]. Ciência Rural, 36(2), 641-647. doi:10.1590/S0103-84782006000 200044.
Martín-Baró, I. (1987). El latino indolente: Carácter ideológico del fatalismo latinoamericano. En M. Montero (Comp.), Psicología Política Latinoamericana [Latin American political psychology] (pp. 135-162). Caracas: Panapo.

Max-Neef, M., Elizalde, A. \& Hopenhayn, M. (1993). Desarrollo a escala humana [Human scale development]. Santiago de Chile: Nordan-Redes.

Méndez Sastoque, M. (2006). Los retos de la extensión ante una nueva y cambiante noción de lo rural [The challenges of extension facing a new and changing notion of rurality]. Revista Facultad Nacional de Agronomía, Medellín, 59, 3407-3423.

Montero, M. (2004). Introducción a la psicología comunitaria: Desarrollo, conceptos y procesos [Introduction to community psychology: Development, concepts and processes]. Buenos Aires: Paidós.

Montero, I. \& León, O. (2007). A guide for naming research studies in Psychology. International Journal of Clinical and Health Psychology, 7(3), 847-862.

Murtagh, S. \& Landini, F. (2011). Producción científica de la psicología vinculada a pequeños productores agropecuarios con énfasis en el ámbito del desarrollo rural [Psychological scientific production related to farmers with emphasis on rural development]. Revista Interamericana de Psicología, 45(2), 293-304.

Oliver, J., Jose, P. \& Brough, P. (2006). Confirmatory factor analysis of the work locus of control scale. Educational and Psychological Measurement, 66(5), 835-851. doi:10.1177/0 013164405285544.

Páez, D. \& Campos, M. (2004). Cultura, evitación de la incertidumbre y confianza interpersonal. En D. Páez, I. Fernández, S. Ubillos \& E. Zubieta (Coords.), Psicología social, cultura y educación [Social psychology, culture and education] (pp. 537-551). Madrid: Pearson. 
Rabbie, J. (1995). Determinantes de la cooperación instrumental intragrupo. En R. Hinde \& J. Groebel (Eds.), Cooperación y conducta prosocial [Cooperation and pro-social behavior] (pp. 263-286). Madrid: Visor.

Sánchez Cadena, D. (2011). Dimensión informativa vs. dimensión comunicativa en el concepto de extensión. ¿La definición según Swanson en verdad ya está superada? [The informative dimension vs. communicative dimension of extension. Is Swanson's definition overcome?]. Ra Ximhai, 7(3), 437-444.

Sánchez Vidal, A. (1991). Psicología comunitaria. Bases conceptuales y operativas, métodos de intervención [Community psychology. Theoretical and operational foundations, intervention metodology]. Barcelona: PPU.

Schaller, N. (2006). Extensión rural: ¿hacia dónde vamos?, ¿hacia dónde ir? [Rural extension: Where are you going? Where should we go?]. El Colorado, Argentina: Ediciones INTA.

Seligman, M. (1989). Indefensión [Helplessness]. Madrid: Debate.

Tomás Sábado, J. (2009). Fundamentos de bioestadística y análisis de datos para enfermería [Foundations for biostatistics and data analysis for nursing]. Barcelona: Universidad Autónoma de Barcelona.

Tsakoumagkos, P., González, M. \& Román, M. (2009). Tecnología y pequeña producción agropecuaria en la Argentina. Una caracterización basada en el Censo Nacional Agropecuario 2002 y en estudios de caso [Technology and small farming in Argentina. A characterization based on the 2002 National Agrarian Census and case studies]. Buenos Aires: Ministerio de Agricultura, Ganadería y Pesca, Argentina.

Valentinuz, C. (2003). La capacitación del productor rural. En R. Thornton \& G. Cimadevilla (Eds.), La extensión rural en debate. Concepciones, retrospectivas, cambios y estrategias para el Mercosur [Rural extension at debate. Approaches, retrospectives, changes and strategies for the Mercosur] (pp. 301-322). Buenos Aires: Ediciones INTA.

Wainstein, M. (2002). Comunicación: Un paradigma de la mente [Communication: A paradigma of the mind]. Buenos Aires: Eudeba.

Watzlawick, P., Beavin, J. \& Jackson, P. (1971). Teoría de la comunicación humana [Pragmatics of human communication]. Buenos Aires: Tiempo Contemporáneo.

Yela Bernabé, J., Gómez, M. \& Salgado Ruiz, A. (2001). Indefensión aprendida: Efectos de la predecibilidad de un estresor crónico [Learned helplessness: Effects of the predictability of a chronic stressor]. Revista Española de Motivación y Emoción, 2, 59-66.

Facultad de Psicología Universidad de Buenos Aires (UBA) Universidad de la Cuenca del Plata Corrientes

República Argentina

Fecha de recepción: 8 de junio de 2012 Fecha de aceptación: 30 de noviembre de 2012 
